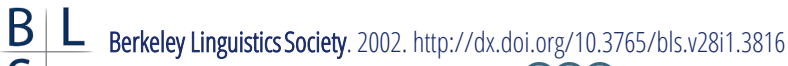

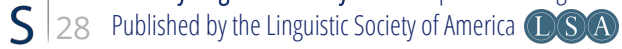

\section{FrameNet's Frames vs. Levin's Verb Classes ${ }^{1}$}

\author{
COLLIN F. BAKER and JOSEF RUPPENHOFER \\ International Computer Science Institute \& University of California, Berkeley
}

\section{Overview}

The classification of verbs in Levin's (1993) English Verb Classes and Alternations: A preliminary Investigation, on the basis of both intuitive semantic grouping and their participation in valence alternations, is often used by the NLP community as evidence of the semantic similarity of verbs (Jing \& McKeown 1998; Lapata \& Brew 1999; Kohl et al. 1998). In this paper, we compare the Levin classification with the work of the FrameNet project (Fillmore \& Baker 2001), where words (not just verbs) are grouped according to the conceptual structures (frames) that underlie them and their combinatorial pattems are inductively derived from corpus evidence. This means that verbs grouped together in FrameNet (FN) might be semantically similar but have different (or no) alternations, and that verbs which share the same alternation might be represented in two different semantic frames.

\section{Basic Comparison}

Table (1) summarizes the two approaches. Note that the numerical comparison of coverage is misleading, because for Levin we are counting distinct lemmas, lumping together senses of polysemous and even homophonous words, but for FrameNet we are counting Lexical Units, which are defined as pairings of lemmas with semantic frames and thus represent separate word senses. (As usual, the problem of dividing and enumerating senses is difficult. Levin says (p. 22) that different senses of a verb will occur in different classes, but this does not always seem to be accurate (cf. Section 7.3).)

\footnotetext{
'We are grateful to the National Science Foundation for funding the work of the FrameNet project through two grants, IRI \#9618838 "Tools for Lexicon Building" March 1997-February 2000, and ITR/HCl \#0086132 "FrameNet++: An On-Line Lexical Semantic Resource and its Application to Speech and Language Technology" September 2000-August 2003. We are also grateful to the Principal Investigator of the FrameNet Project, Charles J. Fillmore, for extensive comments on this paper; any errors that remain are our own.
} 
(1)

\begin{tabular}{|l|l|l|}
\multicolumn{2}{c}{ FrameNet } & Levin 1993 \\
\hline Groupings & 230 semantic frames & 193 verb classes \\
\hline Basis & lexical semantics & argument syntax \\
\hline Data Source & Corpora & linguistic literature \\
\hline Coverage & $\begin{array}{l}\text { 2100 nouns, 1700 verbs } \\
\text { (including multi-word } \\
\text { expressions), 460 adjectives }\end{array}$ & 3100 verbs \\
\hline Results & $\begin{array}{l}\text { Frame descriptions and } \\
\text { annotated examples }\end{array}$ & $\begin{array}{l}\text { Verb classes and alternations } \\
\text { (most with descriptions) }\end{array}$ \\
\hline
\end{tabular}

As the number of categories in the two studies is comparable ( 230 frames vs. 193 verb classes), one might expect that many of the FN frames would correspond to Levin's verb classes, and vice-versa. There are many partial correspondences, but there are many significant differences as well.

\section{Basic Difference in Perspective}

In FrameNet, predicates belong to frames based on a shared semantics. They need not exhibit all the same syntactic behaviors in order to be able to be grouped together. Thus, our frames can include alternators and non-alternators. Consider the verbs load and fill, which have long been central to the discussion of alternations.

(2)

Load, fill, and related verbs in FrameNet and Levin (1993)

\section{FrameNet}

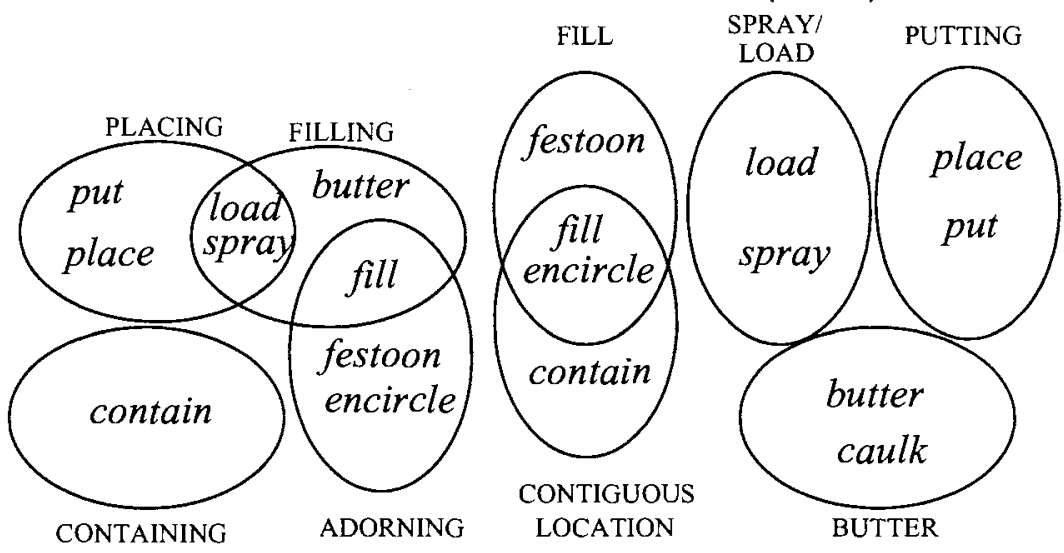

According to FrameNet, fill and load are both in the Filling frame. Load is, additionally, in the Placing frame, while fill is also listed in the Adorning frame. 
This reflects the facts (both syntactic and semantic) that Filling is causative (Theme-Object) and Adorning (Theme-Subject) is not.

Since alternation patterns are criterial in Levin's system, alternators and nonalternators cannot be in the same verb class, and the interchangeability between fill and load in the syntactic pattern exemplified in examples (3) and (4) is therefore not captured. (All examples are from the British National Corpus (BNC) unless otherwise noted.)

(3) While the shop assistant helped another customer and the children played, the 2 adults filled their pockets with rings and other valuables.

(4) . He loaded the barrow with paving stones before running straight through a plate glass window at the B superstore in South Shields.

Likewise, Levin has a separate class for Butter Verbs, noting that they "all have zero-related nominals; their meaning can be paraphrased as 'put $\mathrm{X}$ on/in something"' but are otherwise similar to the Spray/Load Verbs. FrameNet records that, for certain verbs in this frame, information about the Theme is incorporated in the verb's meaning but does not regard the basic semantics as different enough from Filling to justify a separate frame. Figure (2) shows the relevant frames, including a few representative words treated in both sources.

\section{Using Corpus Data}

FrameNet's classifications and lexical entries are based on attested corpus examples. In many of Levin's classes there are certain members for which our corpus data do not support their use by speakers in the constructions that Levin predicts they should occur in. For instance, her Verbs of Instrument of Communication (telex, wire, semaphore, phone, telephone, cable, telegraph, radio, $f a x$ ) are said to be able to occur as parentheticals in indirect quotations, e.g., The winner, Heather cabled (Sara), would be announced tonight. The verb cable, however, is the only one on the list attested in this construction in the BNC.

Let us look in detail at the verb telephone. Corpus study shows that some uses in the BNC match Levin's predictions:

(5) In October 1944, Mr. Argles telephoned the Birmingham office and said that his wife was severely indisposed.

(6) She might have backed off, gone into the pub and telephoned for a cab.

(7) [She] telephoned the young woman's mother to come ...

(8) The following day, Moira telephoned the Daily Telegraph with profuse apologies for the misunderstanding.

(9) ... you should telephone your flight-plan to Lisbon ...

(10) My Chief-of-Staff telephoned to me that the attack had failed and that everywhere our men could be seen falling back.

Other uses are not discussed at all by Levin: 
And some uses predicted by Levin are not attested at all: ${ }^{2}$

(12) ?Mom telephoned me the good news.

(13) ?Mom telephoned me that she was ill.

(14) ??My brother, mom had telephoned me, was now in the hospital.

\section{Comparison of Groupings}

Although the whole thesis of Levin's work is that grouping words according to alternations tends to produce semantically coherent classes, it can also split words that are close in meaning, or lump semantically disparate words. In this section, we will discuss examples of four types of mismatch with FrameNet frames.

\subsection{Levin class roughly equivalent to $\mathrm{FN}$ frame}

(15)

\begin{tabular}{|l|l|}
\hline Levin Cooking Verbs & FrameNet Apply heat Frame \\
\hline bake, barbecue, blanch, boil, braise, broil, & baste, blanch, boil, braise, \\
brown, charbroil, charcoal-broil, coddle, cook, & broil, brown, char, coddle, \\
French fry, fry, grill, hard-boil, heat, microwave, & fry, grill, microwave, parboil, \\
oven-fry, oven-poach, over-cook, pan-broil, pan- & poach, roast, sauté, scald, \\
fry, parboil, parch, perk, plank, poach, pot-roast, & simmer, steam, steep, stew, \\
rissole, roast, sauté, scald, scallop, shirr, & toast,... \\
simmer, steam, stew, stir-fry, toast, ... & \\
\hline
\end{tabular}

Levin defines this class partially on the basis of the three altemations:

$\begin{array}{lll}\text { Causative/ } & \text { Jan is baking the potatoes } & \text { The potatoes are baking } \\ \text { Inchoative } & & \\ \text { Middle } & \text { Jan baked Idaho potatoes } & \text { Idaho potatoes bake beautifully } \\ \text { Instrument } & \begin{array}{l}\text { Jan bakes the potatoes in that } \\ \text { oven }\end{array} & \text { That oven bakes potatoes well } \\ \text { Subject } & \begin{array}{l}\text { Then } \\ \end{array}\end{array}$

However, some of these alternates are rare; for example, of the 142 annotated examples of verbs in the Apply heat frame in which the frame element Food appears, none permit a Middle interpretation. (But we should be cautious in ascribing significance to the number of annotated examples, see below).

\footnotetext{
${ }^{2}$ Some of these may be judged grammatical by some speakers, but they evidently are not found among the 1,200 examples of the verb telephone in the BNC.
} 


\subsection{Levin class narrower than $F N$ frame}

(17)

\begin{tabular}{|l|l|}
\hline Levin (1993) Classes & FrameNet Placing frame \\
\hline Pocket Verbs: archive, bag, bank, ... & archive, bag, bank, mount, place, put, \\
Put Verbs: mount, place, put, ... & $\ldots$ \\
\hline
\end{tabular}

The verbs of Putting and Placing are divided up by Levin into those which are morphologically related to a noun denoting the goal location and others for which this is not the case. FrameNet does not make such a distinction, given that these verbs do not incorporate a referential argument. Rather we assume that the incorporated arguments are interpreted like indefinite null instantiations in the sense of Fillmore 1986. Indeed, cognate location phrases are not categorically ruled out but are possible when more specific information is to be given about the goal location:

(18) The vinegar is then bottled in the traditional flask and sealed with a cork to preserve its high quality.

\subsection{Levin class broader than FN frame}

Reliance on syntactic alternations also leads Levin to posit some very broad, semantically very abstract classes. Examples of this are Levin's verb classes of social interaction (36): correspond, marry, and meet verbs. These three classes are defined syntactically by alternations reflective of the notion of reciprocality:

\begin{tabular}{|l|l|}
\hline Collective subject NP & The committee bantered/met \\
\hline Simple Reciprocal Alternation & $\begin{array}{l}\text { Pat bantered/met/"married with Kim } \\
\text { Pat and Kim bantered/met/married }\end{array}$ \\
\hline Understood Reciprocal Object & $\begin{array}{l}\text { Pat married/met } / * \text { bantered Kim } \\
\text { Pat and Kim bantered/married/met }\end{array}$ \\
\hline
\end{tabular}

However, it appears that the alternations that Levin describes as characteristic of this verb class are not in fact diagnostics of reciprocality. For instance, even when the actions of the participants are not directed at each other but just jointly or simultaneously undertaken, plural (John and Sue jogged), coordinate (John jogged with Sue), or collective subjects (The group jogged) are acceptable. Furthermore, the encoding of one argument slot by a reciprocal is available with events that are not inherently reciprocal (Larry and Moe looked at each other).

Thus, verbs of social interaction, in so far as they are understood as involving reciprocal action of the participants, cannot be identified with the help of the above constructions. Proposing a more general verb class that includes all verbs denoting necessarily or optionally reciprocal events would result in a verb class of enormous size and semantic diversity. Clearly, the investigation in this case has to start from the semantics rather than the syntax. 
Looking now at the semantics of Levin's three proposed subclasses, we find them internally rather heterogeneous.

(20)

\begin{tabular}{|l|l|l|}
\hline Correspond verbs & Marry verbs & Meet verbs \\
\hline agree, argue, banter, bargain, bicker, & court, cuddle, date, & battle, box, \\
brawl, clash, coexist, collaborate, & divorce, embrace, & consult, debate, \\
collide, combat, commiserate, commu- & hug, kiss, marry, & fight, meet, play, \\
nicate, compete, concur, confabulate & muzzle, pass, pet & visit \\
\hline
\end{tabular}

In our treatment of Levin's Correspond verbs, argue, for instance, is assigned to a Communication_conversation frame along with bicker, chat, gossip etc., whereas struggle is placed in the Hostile encounter frame. Similarly, FN puts the verbs in Levin's Marry class (36.2) into different frames. Marry, date, court, divorce belong to the Personal_relationship frame along with other words such as bachelor, boyfriend, break-up, wife, woo. The remaining words in Levin's class are currently not in the FN database but arguably belong to something like a Display_of_affection frame, which would also contain caress, pet (an animal).

Levin's Meet class (36.3) differs syntactically from the other two by allowing for one party to appear as a direct object (Pat met Kim) as well as for it to appear in a with-phrase (Pat met with $\mathrm{Kim}$ ). However, it seems to us that the verbs Levin puts in this class do not have the same meaning in the transitive and the with-PP use. For instance, box in I ended up boxing with him does not necessarily involve a competition in the same way as Tyson will box Lewis. Meaning differences exist also with play and meet (My son played/met with your son $\neq$ My son played/met your son). Thus, the Meet class is not a genuine separate class from the other two and the pairs of senses in the Meet class can be distributed to other frames.

Note finally that FrameNet would not put the verbs that are only optionally reciprocal into separate frames in those uses. For these cases, we would rely on mechanisms of semantic composition to yield the right kinds of interpretation.

\subsection{Overlapping Groupings: Communication verbs}

The verbs of communication, like the spray/load/fill verbs discussed above, show a more complex sort of overlapping of FrameNet and Levin classes.

Communication verbs are one area in which the strategy of grouping by a verb's unique set of altemations leads to overly narrow classes. In the case of the verb tell, for instance, Levin, unlike FrameNet, does not distinguish the 'order' sense in (21), where tell takes a VP ${ }_{t o}$ complement, from the 'inform/say to' sense in (22a-b), where it takes NPs and finite clauses as a complement:

(21) Maybe the French told her to act pregnant and so lengthen her stay in Scotland!

(22) a. I tell you I'm not satisfied with that pesky voyage.

b. "Yes, I told her my theory," he said under his breath. 
Similarly, Levin groups speak and talk into a unique class, whereas FrameNet groups the conversational uses in (23) with those of verbs like chat and argue, and the statement-like use of speak in (24) with those of announce, claim etc.

a. He had spoken with Amelie who, though still incapacitated with her broken hip, was desperate to see Peach.

b. Would you like some coffee and then we could talk?

'He seems very nice,' Emily spoke guardedly, 'but what of his prospects?'

The two groupings are summarized in Figures (25) and (26). For Levin, the alternations dictate that tell be in a class by itself and that speak and talk be in a separate class. From our point of view, these are lexical idiosyncrasies within the semantic groupings.

(25) Communication verbs in Levin

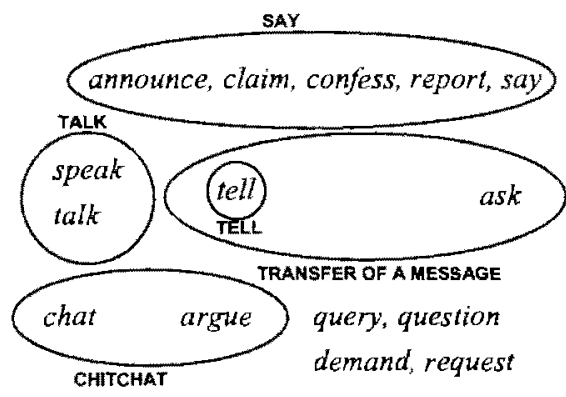

(26) Communication verbs in FrameNet

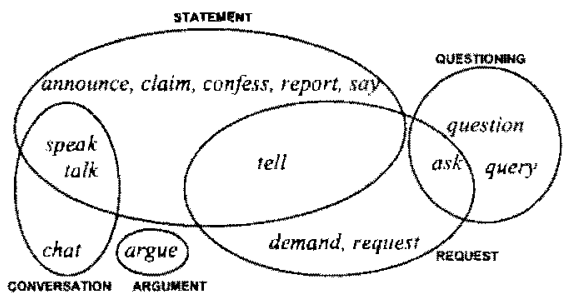

\section{Semantically meaningful Levin classes?}

Levin says that "verbs in English and other languages fall into classes on the basis of shared components of meaning. The class members have in common a range of properties, including the possible expression and interpretation of their arguments, as well as the existence of certain morphologically related forms." But the meaning which is to be associated with a Levin class is often hard to define. As Dang et al. (1998) observe, "Of course, some Levin classes, such as braid (bob, braid, brush, clip, coldcream, comb, condition, crimp, crop, curl, etc.) are clearly not intended to be synonymous." In addition, many verbs are cross-listed in classes which pick out one aspect of their meaning but do not capture separate senses. An example of this are the hundreds of verbs found in Other Altemating Verbs of Change of State. The FN frame hierarchy allows us a more appropriate level of generalization for the facts relating to change-of-state verbs (see below).

The commitment to define separate classes of words according to their morphological make-up also causes Levin to make decisions differently from FrameNet. Thus, FrameNet could include call (i.e. on the telephone) and write in 
the Communication_means frame, but Levin would not, since writing is not zerorelated, and a call is not an instrument.

We saw earlier that verbs of Instrument of Communication varied amongst themselves in respect to their valence, especially as found in the corpus, and we can see that call and write exhibit some of those same patterns, but not others. And in the same way that some of Levin's verbs have related result nouns (wire and cable but not phone or telephone), call has a result noun meaning but write does not. (We received several important cables/wires/calls/*writes $/{ }^{*}$ writings.) Likewise, the commitment to syntactically defined alternations requires Levin to list, e.g. track twice, both in the Stalk verbs (35.3) and the Chase verbs $(51.6)$; most people would probably consider this one sense, rather than two.

\section{Comparison of Hierarchies}

Levin's (1993) list of verb classes is divided into 51 sections, with two further levels of subdivision. The sections

reflect a limited attempt to group verb classes related by meaning together. However, there is little hierarchical organization compared to the number of classes identified. This lack of structure reflects not only the preliminary nature of the investigation, but also the fact that it is an open research question whether a complete hierarchical organization of English verbs is possible or even desirable (see Fellbaum 1990 and Miller \& Fellbaum 1991 for some discussion)" (Levin 1993:23).

As an example, Figure (27) shows the semantic relations indicated by Levin's subclasses of the Communication verbs, while Figure (28) shows the FrameNet inheritance structure in this area.

Relations among Levin's verb classes

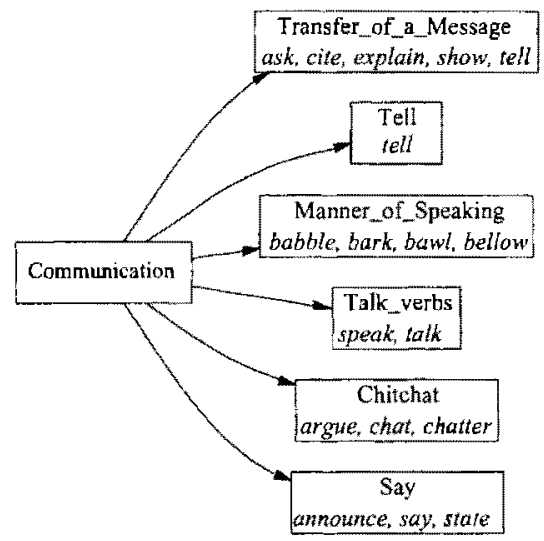


Not only does FN define inheritance lattices relating frames, but we have a parallel, detailed set of inheritance relationships among their Frame Elements. (These are not illustrated here because the graph is quite complex.) Although the FN inheritance relations do not yet connect all frames into larger structures and although it is not clear what form such larger structure(s) might take, multiple inheritance should allow us a fuller representation of the relations among frames.

Relations among FrameNet frames

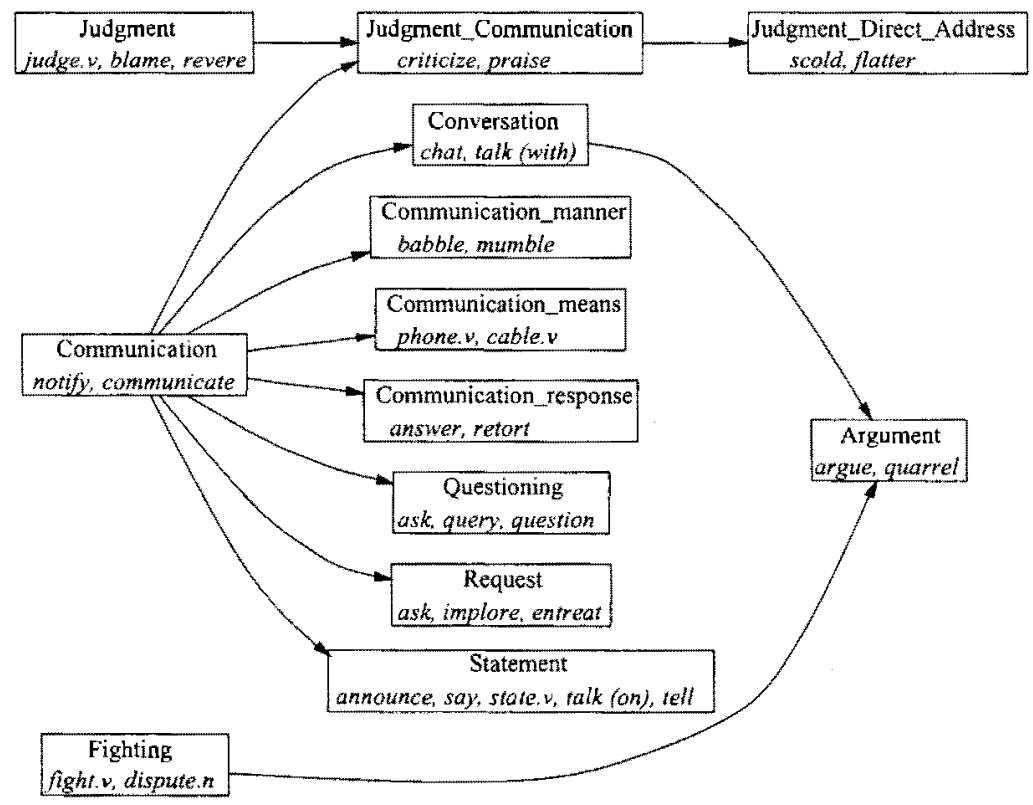

\section{Challenges for both treatments}

\subsection{Alternations not related to lexical semantics of the verb}

It is important to notice that neither Levin nor FrameNet assume that all verb syntax reflects only the inherent semantics of the verb. Of course, some alternations do reflect the underlying lexical semantics. An example is the altemation between the conative and the simple transitive construction, which differentiates, e.g., the verbs bite and break. Both verbs can occur in the transitive construction. Bite can also occur in the conative construction (The dog bit at Sue) since it does not lexicalize success in biting, only the attempt. By contrast, break cannot be used this way ( ${ }^{*}$ Bill broke at the vase) since it lexicalizes a completed change of state. Levin depends on this alternation in many decisions about grouping. 
Two constructions that do not seem to relate to inherent lexical meaning in the same way are Locative lnversion and There-insertion, shown in (29) - (31):

(29) Into the room came Harry. (Bresnan)

(30) From the speakers drones the voice of Max Von Sydow. (BNC)

(31) Out of this blur there stares a single set of eyes. (BNC)

As pointed out in much of the syntactic and discourse-pragmatic literature, these constructions are used when new referents are to be introduced into the discourse. However, we do not want to posit an 'appearance/existence' sense for all the verbs that can occur in them. Rather, we assume that the semantics of verbs belonging to frames such as Perception_noise (drone), Perception_active (stare), and Self motion (come) is compatible with the discourse-pragmatic function of introducing new referents. For instance, by having only one participant, these verbs allow speakers to introduce a new referent in one clause before predicating about it in the next clause, which is the preferred discourse strategy. In short, not all argument syntax reflects lexical semantics in a narrow sense.

\subsection{Frequency Data}

Currently, neither approach incorporates information about the frequency of a verb's syntactic patterns in text genres. While the relevance of probabilistic knowledge is contested in the field of theoretical linguistics (despite some influence from experimental studies such as Schuetze (1996) and from the emerging field of corpus linguistics), it is clearly a useful notion for NLP (e.g., Manning (1993)).

It is important to recognize that, although one can count the annotated examples in FrameNet, they cannot be taken as representative of the frequencies of FEs or valence patterns in running text. FrameNet annotations exist for the purpose of documenting the range of syntactic possibilities for lexicographic purposes. Research on the real frequencies of FEs and their valence patterns in running text is being carried out by our associates at the University of Colorado (e.g., Roland et al. (2000)).

\subsection{Relationships between senses}

Another theoretical limitation of both the FrameNet approach and the Intersective Classes approach of Dang et al. (1998) is that relationships between senses of words can be characterized only by positing a more general sense that relates the two more specific ones. (Levin (1993) does not discuss this issue.) In FrameNet this is done by inheritance; in the intersective class approach, by intersection. Neither treatment is adequate for all types of sense relations. For instance, the uses shown in (32) - (35) of a word like argue belong to two different frames, Evidence and Statement, whose relationship is not discussed explicitly by FrameNet. 
(32) ... all of which argues that only a small minority of policemen and women define their work in terms of social service.

(33) Seurat and his colleagues argued that Pointillism was a scientifically modern style,

(34) *The facts clearly announce that we should do it this way.

(35) ?The spokesperson substantiates that the company will build the plant in Pittsburgh.

One solution to this problem would be to remove the words from Statement and move them into a frame that inherits from both Statement and Evidence. This is an appealing solution, since both the notions of evidence and of communicating are simultaneously required. However, the inheritance solution would run counter to the intuition that the speaking sense of words like argue is more basic than the evidential one; the same intuition also holds for other word doublets: My brother persuaded me to drive more slowly versus $M y$ accident persuaded me to drive more slowly. But there are differences among words in these frames in regard to this polysemy, as shown by examples (34) and (35).

\section{Conclusions}

Levin (1993) demonstrated that syntactic alternations can be the basis for groupings of verbs that make some semantic sense, and that accord reasonably well with linguistic intuitions. Detailed examination of Levin's classes and altemations, as in Dang et al. (1998), reveals that (a) the classes are not simply the product of automatic application of a set of rules about participation in alternations, but are partially semantically motivated, and (b) a classification rigorously and solely based on alternations would give much finer distinctions, including splitting of many semantically coherent classes. The FrameNet project is producing a lexicon with roughly comparable coverage of verbs, but with much more detail concerning the semantics and syntax of their arguments, more semantically consistent categories, and a richer set of relations among them. The FrameNet lexicon also covers nouns and adjectives, using the same semantic frames.

\section{References}

Bresnan, Joan. 1994. Locative inversion and the architecture of universal grammar. Language. 70:72-131.

Chafe, Wallace. 1987. Cognitive constraints on information flow. In Russell Tomlin (ed.) Coherence and Grounding in Discourse, 21-51. Amsterdam: John Benjamins.

Dang, Trang Hoa, Karen Kipper, Martha Palmer, \& Joseph Rosenzweig. 1998. Investigating regular sense extensions based on intersective Levin classes. Proceedings of COLING-ACL. Montréal. 293-99. 
Fellbaum, Christiane. 1990. English verbs as a semantic net. International Journal of Lexicography. 3:278-301.

Fillmore, Charles, \& Collin F. Baker. 2001. Frame semantics for text understanding. Proceedings of WordNet and Other Lexical Resources Workshop. NAACL. Pittsburgh. 59-64.

Jing, Hogyan, \& Kathleen McKeown. 1998. Combining multiple, large-scale resources in a reusable lexicon for natural language generation. Proceedings of COLING-ACL. Montréal. 607-13.

Kohl, Karen T., Douglas A. Jones, Robert C. Berwick, \& Naoyuki Nomura. 1998. Representing verb alternations in WordNet. In Christiane Fellbaum (ed.) WordNet: An Electronic Lexical Database, 153-178. Language, Speech, and Communication. Cambridge: MIT Press.

Lakoff, George. 1987. Women, Fire, and Dangerous Things. Chicago: University of Chicago Press.

Lambrecht, Knud. 1994. Information Structure and Sentence Form. New York: Cambridge University Press.

Lapata, Maria, \& Chris Brew. 1999. Using subcategorization to resolve verb class ambiguity. Proceedings of the 1999 Joint SIGDAT Conference on Empirical Methods in Natural Language Processing and Very Large Corpora. College Park, MD. 266-74.

Levin, Beth. 1993. English Verb Class and Alternations: A Preliminary Investigation. Chicago: University of Chicago Press.

Manning, Christopher. 1993. Automatic acquisition of a large subcategorization dictionary from corpora. Proceedings of the $3 I^{\text {st }}$ Annual Meeting of the Association for Computational Linguistics. 235-42.

Miller, George A., \& Christiane Fellbaurn. 1991. Semantic networks of English. Cognition. 41:197-229.

Roland, Douglas, Daniel Jurafsky, Lise Menn, Susanne Gahl, Elizabeth Elder, \& Chris Riddoch. 2000. Verb subcategorization frequency differences between business-news and balanced corpora: the role of verb sense. Proceedings of the Association for Computational Linguistics Workshop on Comparing Corpora.

Schütze, Carson T. 1996. The Empirical Base of Linguistics: Grammaticality Judgments and Linguistic Methodology. Chicago: University of Chicago Press.

Collin F. Baker \& Josef Ruppenhofer

International Computer Science Institute

1947 Center St., Suite 600

Berkeley, California 94704

collinb@icsi.berkeley.edu

josef@icsi.berkeley.edu 